\title{
ALEXANDER REPRESENTATION OF TANGLES
}

\author{
STEPHEN BIGELOW, ALESSIA CATTABRIGA, AND VINCENT FLORENS
}

\begin{abstract}
A tangle is an oriented 1-submanifold of the cylinder whose endpoints lie on the two disks in the boundary of the cylinder. Using an algebraic tool developed by Lescop, we extend the Burau representation of braids to a functor from the category of oriented tangles to the category of $\mathbb{Z}\left[t, t^{-1}\right]$ modules. For (1,1)-tangles (i.e., tangles with one endpoint on each disk) this invariant coincides with the Alexander polynomial of the link obtained by taking the closure of the tangle. We use the notion of plat position of a tangle to give a constructive proof of invariance in this case.
\end{abstract}

\section{INTRODUCTION}

The Burau representation was the first non trivial representation of the braid groups. It was defined by writing an explicit matrix in $G L_{n}\left(\mathbb{Z}\left[t, t^{-1}\right]\right)$ for every generator of $B_{n}$, and verifying that these matrices satisfy the braid relations. Later, it was reinterpreted with the point of view of mapping class group and action on Burau modules, which are homology modules of the punctured disk with twisted coefficients in $\mathbb{Z}\left[t, t^{-1}\right]$, as a particular case of the Magnus representation [B1]. This representation was also the first endowed with a unitary structure, and extensively studied for its (non)-faithfulness.

Several extensions of the Burau representation have been considered. The first was due to Le Dimet [Ld for string-links, and studied in more detail by Kirk and Livingston [KLW. An extension for the tangle category was defined by Cimasoni and Turaev [CT1, CT2]; it takes value in the Lagrangian category, which contains that of $\mathbb{Z}\left[t, t^{-1}\right]$-modules.

An important aspect of braid group representations is their connection with polynomial invariants of knots. One reconstructs the Alexander polynomial of a closed braid from the Burau representation as $\operatorname{det}(i d-\rho)$. The other works on the extension of the representation to string links or tangles contain some relations with the Alexander polynomial, but no direct formula.

In this paper we use an algebraic tool introduced by Lescop for link complements to construct an isotopy invariant for oriented tangles. This invariant is related to the Alexander module of the tangle exterior in the cylinder. This approach is functorial: the invariant provides a functor from the category of oriented tangles to the category of $\mathbb{Z}\left[t, t^{-1}\right]$-modules and homomorphisms up to multiplication by a unit and for $(1,1)$-tangles, it coincides with the Alexander polynomial of the closure of the tangle. Our construction is a particular case of a more general construction developed in [FM].

Section 2 is devoted to the construction of the invariant. In Section 3 we compute the value of the invariant for the generators of the category. In Section 4 the

2010 Mathematics Subject Classification. Primary 57M27; Secondary 57M25. 
invariant is computed explicitly when the tangle is written as the plat closure of an oriented braid. This illustrates the similarities with the approach of Lawrence La] for the Jones polynomial and with the model of Bigelow for a link presented as a plat closure. In the last Section we prove the functoriality of the invariant.

It should be noted that the homology of symmetric spaces of the punctured disk (or configuration spaces), with some twisted coefficients coincides with the exterior algebra of the Burau modules. The modules can also be endowed with a Hermitian structure coming from the exterior power of the twisted intersection form. In particular, for $(1,1)$-tangles, the calculation could be interpreted as an intersection (with twisted coefficients) of a set of curves in the 2-disk.

It is worth mentioning that the model of Bigelow for the Jones polynomial inspired some construction of categorification. Our approach here should similarly give rise to a categorification of the Alexander polynomial - presumably some form of Heegaard Floer homology.

\section{The MAIN CONSTRUCtion}

2.1. The Alexander functions of a module. Let $R=\mathbb{Z}\left[t, t^{-1}\right]$ be the Laurent polynomial ring. Let $H$ be an $R$-module of finite type. Consider a free resolution of $H$ of the form

$$
R^{p} \stackrel{A}{\longrightarrow} R^{p+k} \longrightarrow H \longrightarrow 0 .
$$

In other words, (11) determines a $k$-deficiency presentation of $H$ over $R$ with $(p+k)$ generators $\gamma_{1}, \ldots, \gamma_{p+k}$ and $p$ relators $\rho_{1}, \ldots, \rho_{p}$. Given such a presentation, it is possible to define an $R$-linear map $\wedge^{k} H \longrightarrow R$ as follows (see [Le, §3.1]).

Let $\hat{\rho}=\rho_{1} \wedge \cdots \wedge \rho_{p}$ and $\hat{\gamma}=\gamma_{1} \wedge \cdots \wedge \gamma_{p+k}$. The Alexander function of $H$ relative to $k$ is the $R$-linear map $\varphi=\varphi(H, k): \wedge^{k} H \rightarrow R$ defined by

$$
u \wedge \hat{\rho}=\varphi(u) \cdot \hat{\gamma}
$$

for each $u \in \wedge^{k} H$. For fixed $k$, different $k$-deficient presentations will give rise to Alexander functions that differ only by multiplication by a unit in $R$.

Note that if $\hat{u}=u_{1} \wedge \cdots \wedge u_{k}$ and $A \in \mathbf{M}_{p \times(p+k)}(R)$ is the matrix associated to the presentation (11), then $\varphi(\hat{u})$ coincides with the determinant of the matrix constructed adding to $A$ the $k$ columns $u_{1}, \ldots, u_{k}$ expressed in terms of the generators $\gamma_{i}$.

Remark 1. From (1), by tensoring with $\mathbb{Q}(t)$, we obtain a presentation of deficiency $k$ for $H \otimes \mathbb{Q}(t)$. So, if $k<\operatorname{rank} H=\operatorname{dim}_{\mathbb{Q}(t)} H \otimes \mathbb{Q}(t)$, then $\varphi$ is the zero map. Moreover, if $H$ is free of rank $k$, then $\varphi$ coincides with the volume form

$$
\wedge^{k} H \stackrel{\cong}{\longrightarrow} R
$$

induced by the choice of $\hat{\gamma}$ as a basis of $\wedge^{k} H$, i.e., $\varphi\left(\gamma_{1} \wedge \cdots \wedge \gamma_{k}\right)=1$.

2.2. The Alexander invariant of a tangle. Let $D$ be the closed unitary disk in $\mathbb{C}$. Consider two copies of the disk $D$ with fixed ordered finite sequences of points $p_{0}, \ldots, p_{k_{-}}$(respectively $p_{0}, \ldots, p_{k_{+}}$) on the real line. Let $\varepsilon^{-}$and $\varepsilon^{+}$be sequences of signs \pm 1 of length $k_{-}+1$ and $k_{+}+1$, respectively. An $\left(\varepsilon^{-}, \varepsilon^{+}\right)$-tangle is an oriented 1-submanifold $\tau$ of $D \times I$ whose oriented boundary $\partial \tau$ is

$$
\sum_{i=0}^{k_{+}} \varepsilon_{i}^{+}\left(p_{i}, 1\right)-\sum_{i=0}^{k_{-}} \varepsilon_{i}^{-}\left(p_{i}, 0\right) \text {. }
$$


Note that if such a tangle exists then $\sum_{i=1}^{k_{+}} \varepsilon_{i}^{+}=\sum_{i=1}^{k_{-}} \varepsilon_{i}^{-}$.

Let $D_{-}$and $D_{+}$be the punctured disks $(D \times\{0\}) \backslash \tau$ and $(D \times\{1\}) \backslash \tau$, respectively. Any sequences of signs $\varepsilon^{ \pm}$determine epimorphisms $\chi_{ \pm}: \pi_{1}\left(D_{ \pm}, *\right) \rightarrow \mathbb{Z}=\langle t\rangle$ sending a simple loop $x_{i}$ turning once around $p_{i}$ in the counterclockwise direction to $t^{\epsilon_{i}^{ \pm}}$. Let $H_{ \pm}$be the $R$-module $H_{1}^{\chi \pm}\left(D_{ \pm} ; R\right)$, where the coefficients are twisted by $\chi_{ \pm}$. Equivalently, $H_{ \pm}$is the first homology of the infinite cyclic covering induced by $\chi_{ \pm}$.

Remark 2. The disk $D_{ \pm}$deformation retracts to the wedge of circles $x_{0} \ldots x_{k_{ \pm}}$. So the $R$-module $H_{ \pm}$is freely generated by $u_{i}^{ \pm}=\tilde{x}_{i}-\tilde{x}_{i+1}$, for $i=0, \ldots, k_{ \pm}$, where $\tilde{x}_{i}$ is a lift of $x_{i}$. More precisely, $u_{i}^{ \pm}$will be a loop around both $p_{i}$ and $p_{i+1}$ if these punctures have opposite signs, or a figure eight around them if they have the same sign.

Let $B_{\tau}$ be the exterior of $\tau$ in $D \times I$. For each connected component $\tau_{j}$ of $\tau$, let $m_{j}$ be a meridian around $\tau_{j}$ oriented so that its linking number with $\tau_{j}$ is one.

Since $H_{1}\left(B_{\tau}\right)=\oplus_{j=1}^{n} \mathbb{Z} m_{j}$ (see [CT1, §3.3]), the composition of the Hurewicz homomorphism with the homomorphism $H_{1}\left(B_{\tau}\right) \rightarrow \mathbb{Z}\langle t\rangle$ sending $m_{i}$ to $t$, gives an epimorphism $\chi: \pi_{1}\left(B_{\tau}\right) \rightarrow \mathbb{Z}$ extending both $\chi_{+}$and $\chi_{-}$. We set $H=H_{1}^{\chi}\left(B_{\tau} ; R\right)$ and denote with $i_{ \pm}$the maps from $H_{ \pm}$to $H$, induced by the inclusion.

Let $\delta k=\frac{k_{+}-k_{-}}{2}$. Our aim is to construct linear maps $\rho_{\tau, \star}$, of degree $\delta k$, between the $R$-modules $\wedge^{\star} H_{-}$and $\wedge^{\star} H_{+}$.

For any generator $w_{+}$of $\wedge^{k_{+}} H_{+} \simeq R w_{+}$, and $r$ with $0 \leq r \leq k_{+}$, consider the isomorphism,

$$
d_{r}: \wedge^{r} H_{+} \longrightarrow \operatorname{Hom}\left(\wedge^{k_{+}-r} H_{+} ; R\right),
$$

defined by the formula $x \wedge y=\left(d_{r}(x)(y)\right) w_{+}$, for any $x \in \wedge^{r} H_{+}$and $y \in \wedge^{k_{+}-r} H_{+}$.

Let $k=\frac{k_{-}+k_{+}}{2}$. We will show in Section 4 that there exists a free deficiency $k$ resolution for $H$ (see Lemma 4.1). For any $i$ with $0 \leq i \leq k$, the choice of generators $\hat{\rho}$ for $\wedge^{p} R^{p}$ and $\hat{\gamma}$ for $\wedge^{k+p} R^{k+p}$ similarly induces isomorphisms

$$
\varphi_{i}: \wedge^{i} H \longrightarrow \operatorname{Hom}\left(\wedge^{k-i} H ; R\right)
$$

defined by the formula $x \wedge y \wedge \hat{\rho}=\left(\varphi_{i}(x)(y)\right) \hat{\gamma}$ in $\wedge^{k+p} R^{k+p}$, for any $x \in \wedge^{i} H$ and $y \in \wedge^{k-i} H$ (see Section 2.1).

Definition 1. For all $i \in\{0, \ldots, k\}$, let the homomorphism $\rho_{\tau, i}: \wedge^{i} H_{-} \rightarrow$ $\wedge^{i+\delta k} H_{+}$be defined by the following composition

$$
\wedge^{i} H_{-} \stackrel{\wedge^{i} i_{-}}{\longrightarrow} \wedge^{i} H \stackrel{\varphi_{i}}{\longrightarrow} \operatorname{Hom}\left(\wedge^{k-i} H ; R\right) \stackrel{\left(\wedge^{k-i} i_{+}\right)^{*}}{\longrightarrow} \operatorname{Hom}\left(\wedge^{k-i} H_{+} ; R\right) \stackrel{d_{i+\delta k}^{-1}}{\longrightarrow} \wedge^{i+\delta k} H_{+} .
$$

The map $\rho_{\tau}=\oplus_{i} \rho_{\tau, i}$ is an isotopy invariant of $\tau$, defined up to a global multiplication by a unit of $R$. We call it the Alexander invariant of $\tau$.

The special case of $(1,1)$-tangles, corresponding to $k_{-}=k_{+}=0$, illustrates the relation with the Alexander polynomial.

Theorem 2.1. Let $\tau$ be a $(1,1)$-tangle and let $\hat{\tau}$ be the link obtained by closing up together the two free strands of $\tau$. Then $\rho_{\tau}$ is equal to $m_{\Delta(\hat{\tau})}$ where $\Delta(\hat{\tau})$ is the Alexander polynomial of $\hat{\tau}$ and $m_{u}: \wedge^{k} H_{-} \rightarrow \wedge^{k} H_{+}$denotes the multiplication by $u$. 
Proof. Since $k_{-}=k_{+}=0$, it follows that $H_{ \pm}=\{0\}$ and $\wedge^{0}\{0\}=R$. Hence $\rho_{\tau}$ is a homomorphism $R \rightarrow R$ that is the multiplication by the determinant of a square presentation matrix of $H$. By definition, this determinant is the Alexander polynomial of $\hat{\tau}$.

\section{The TANGLE CATEGORY}

Let $\mathcal{T}$ denote the oriented tangle category, that is, the category whose objects are sequences $\epsilon=\left(\epsilon_{0}, \ldots, \epsilon_{n}\right)$ of signs \pm attached to the punctures of a punctured disk, and whose morphisms are oriented tangles.

Let $\mathcal{M}$ be the category whose objects are $R$-modules and whose morphisms are classes of homomorphisms modulo the equivalence relations defined by $f \sim g$ if there exists a unit $u \in R$ such that $f=m_{u} \circ g$ where $m_{u}$ denotes the multiplication by $\mathrm{u}$. There is a functor from $\mathcal{T}$ to $\mathcal{M}$ taking $\epsilon^{-} \stackrel{\tau}{\longrightarrow} \epsilon^{+}$to the class of modules homomorphisms $H_{-}\left(D_{\epsilon^{-}}\right) \stackrel{\rho_{\tau}}{\longrightarrow} H_{+}\left(D_{\epsilon^{+}}\right)$. We postpone the proof of this statement to last section, and, in the following, we compute $\rho_{\tau}$ on the generators of the tangle category.

3.1. Braids. Let us start with an oriented identity braid $\tau$ with $k+1$ strands. Thus $H$ is free of rank $k$, and $\varphi: \wedge^{k} H \rightarrow R$ is an isomorphism and coincides with the volume form induced by the choice of a basis. Moreover, $i_{-}$and $i_{+}$are isomorphisms, and the choice of det+ induces a canonical choice of $\varphi$, such that the following diagram commutes.

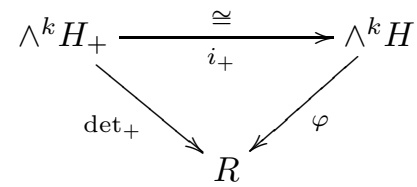

Let $u_{-}$be in $\wedge^{i} H_{-}$. From the diagram, for all $w_{+} \in \wedge^{k-i} H_{+}$, we get

$$
\operatorname{det}_{+}\left(i_{+}^{-1}\left(i_{-}\left(u_{-}\right) \wedge i_{+}\left(w_{+}\right)\right)=\varphi\left(i_{-}\left(u_{-}\right) \wedge i_{+}\left(w_{+}\right)\right) .\right.
$$

By definition,

$$
\varphi\left(i_{-}\left(u_{-}\right) \wedge i_{+}\left(w_{+}\right)\right)=\operatorname{det}_{+}\left(\rho_{\tau}\left(u_{-}\right) \wedge w_{+}\right) .
$$

Since det $_{+}$is non singular, we get

$$
\rho_{\tau}\left(u_{-}\right)=i_{+}^{-1}\left(i_{-}\left(u_{-}\right)\right) .
$$

Now consider the general case of an oriented braid $\sigma$ in $B_{k+1}$, i.e., $\sigma$ is the isotopy class of a homeomorphism of the $(k+1)$-punctured disk (that we still denote with $\sigma)$. The geometric realisation of $\sigma$ as a braid with $(k+1)$-strings in $D \times I$ can be viewed as the mapping cylinder of $\sigma$. Let $\left\{u_{1}^{ \pm}, \ldots, u_{k_{ \pm}}^{ \pm}\right\}$be the the basis of $H_{ \pm}$described in Remark 2, Since $i_{-}: H_{-} \rightarrow H$ is still an isomorphism we can choose $\left\{i_{-}\left(u_{1}^{-}\right), \ldots, i_{-}\left(u_{k_{-}}^{-}\right)\right\}$as a free basis for $H$. With respect to these bases, the matrix associated to $i_{+}$is $b(\sigma)^{-1}$, where $b(\sigma)$ is the image of $\sigma$ by the oriented Burau representation. It follows that, with respect to these bases the matrix associated to $\left(\rho_{\tau}\right)_{i}: \wedge^{i} H \rightarrow \wedge^{i} H_{+}$is $\operatorname{det}(b(\sigma))\left(\wedge^{i}\left(b(\sigma)^{-1}\right)\right)$. Moreover, the 
matrices of the oriented Burau representation of the Artin generators $\sigma_{1}, \ldots, \sigma_{k-1}$ of $B_{k+1}$ in the bases $u_{1}^{ \pm}, \ldots, u_{k_{ \pm}}^{ \pm}$are

$$
\begin{gathered}
M_{b\left(\sigma_{1}\right)}=\left(\begin{array}{cc}
-t^{\varepsilon_{2}} & 1 \\
0 & 1
\end{array}\right) \oplus I d_{k-3}, M_{b\left(\sigma_{k-1}\right)}=I d_{k-3} \oplus\left(\begin{array}{cc}
1 & 0 \\
t^{\varepsilon_{k}} & -t^{\varepsilon_{k}}
\end{array}\right) \\
M_{b\left(\sigma_{i}\right)}=I d_{i-2} \oplus\left(\begin{array}{ccc}
1 & 0 & 0 \\
t^{\varepsilon_{i+1}} & -t^{\varepsilon_{i+1}} & 1 \\
0 & 0 & 1
\end{array}\right) \oplus I d_{k-i-2} .
\end{gathered}
$$

3.2. A cup. Let cup be the tangle in Figure1. We have $k_{-}=k_{+}-2, k=k_{+}-1$, $\delta k=1$ and $H$ free. Note that $i_{-}$is a monomorphism and we can choose the bases of $H_{-}$and $H$, such that the matrix for $i_{-}$is $I_{k_{-}} \oplus 0$. On the other hand, $i_{+}$is an epimorphism and the kernel of $i_{+}$has rank one. Using Mayer-Vietoris arguments on a small disk around the points $p_{k+1}$ and $p_{k+2}$ in $D_{+}$, we can identify the basis of $H$ with a sub-basis of $H_{+}$so that $i_{+}$is simply a projection.

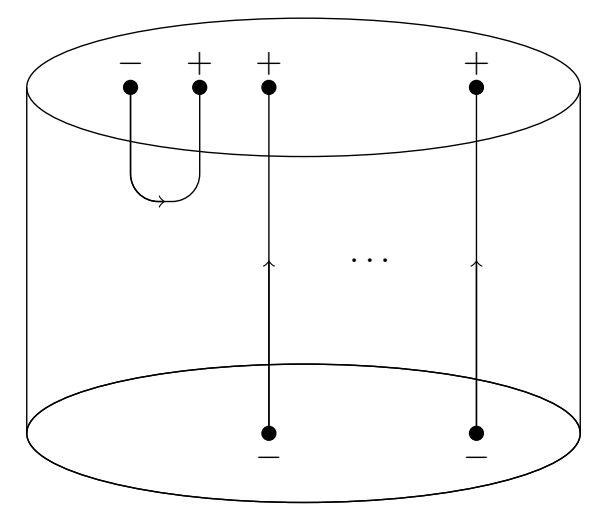

Figure 1. A cup.

Let $\alpha \in H_{+}$be a generator of the kernel of $i_{+}$. Consider the contraction along $\alpha$, that is

$$
i_{\alpha}: \operatorname{Hom}\left(\wedge^{k} H_{+}, R\right) \rightarrow \operatorname{Hom}\left(\wedge^{k_{+}-1} H_{+}, R\right)
$$

such that $i_{\alpha}\left(\operatorname{det}_{+}\right)\left(v_{+}\right)=\operatorname{det}_{+}\left(v_{+} \wedge \alpha\right)$, for any $v_{+} \in \wedge^{k_{+}-1} H_{+}$. In other words, the following diagram commutes.

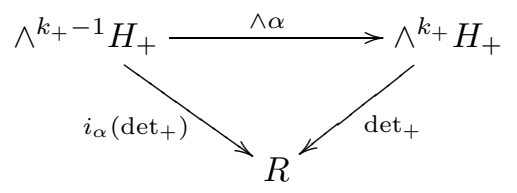

Since $\alpha \in \operatorname{ker} i_{+}$, one has the following.

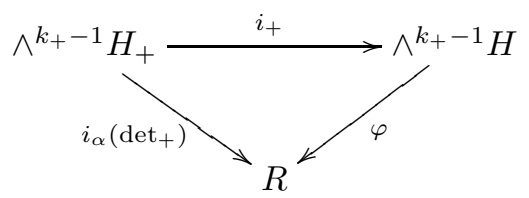


Let $u_{-}$be in $\wedge^{i} H_{-}$, for $i=1, \ldots, k$. By definition, for all $w_{+} \in \wedge^{k-i} H_{+}$,

$$
\operatorname{det}_{+}\left(\rho_{\tau}\left(u_{-}\right) \wedge w_{+}\right)=\varphi\left(i_{-}\left(u_{-}\right) \wedge i_{+}\left(w_{+}\right)\right) .
$$

By the previous diagrams,

$$
\varphi\left(i_{-}\left(u_{-}\right) \wedge i_{+}\left(w_{+}\right)\right)=\operatorname{det}_{+}\left(i_{+}^{-1} i_{-}\left(u_{-}\right) \wedge w_{+} \wedge \alpha\right)
$$

where by $i_{+}^{-1} i_{-}\left(u_{-}\right)$we mean any preimage of $i_{-}\left(u_{-}\right)$, since two of them differ by addition of a multiple of $\alpha$. From above, since det + is non-singular, we get

$$
\rho_{\tau}\left(u_{-}\right)=(-1)^{k-i} i_{+}^{-1} i_{-}\left(u_{-}\right) \wedge \alpha
$$

3.3. A cap. Let cap be the tangle in Figure 2. We have $k_{-}=k_{+}+2, k=k_{+}+1$, $\delta k=-1$ and $H$ free. Here $i_{+}$is a monomorphism. We write $H=i_{+}\left(H_{+}\right) \oplus \beta$. Similarly to the previous section, we can define $i_{\beta}$ the contraction along $\beta$ and we have the following commutative diagrams.
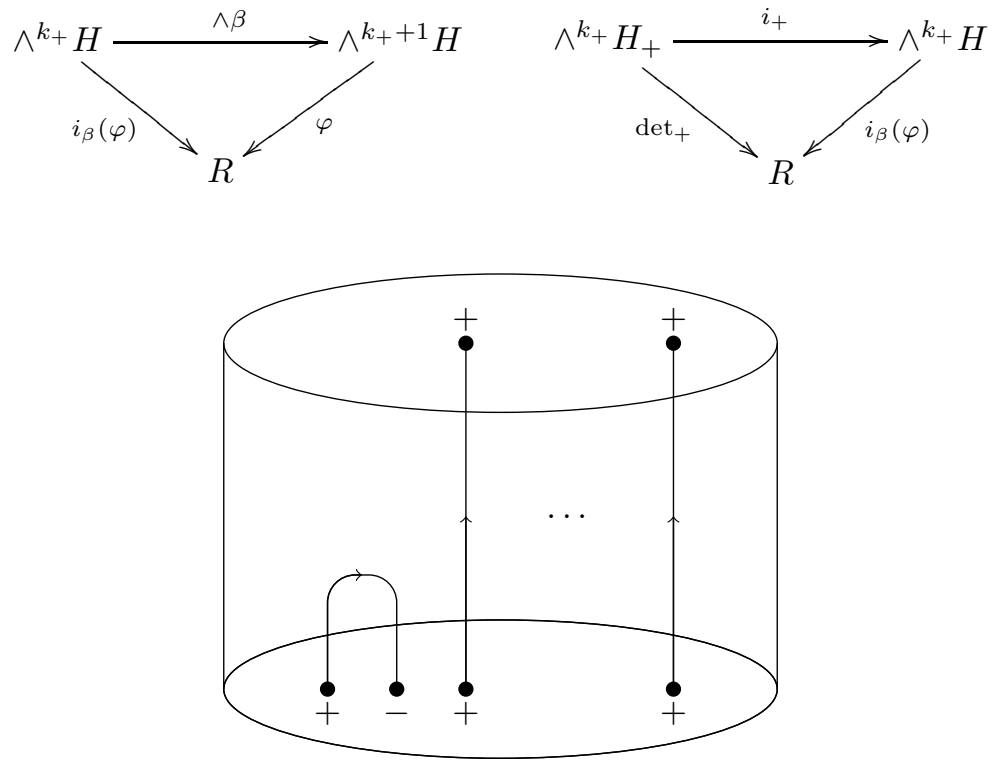

Figure 2. A cap.

Let $u_{-}$be in $\wedge^{i} H_{-}$. For all $w_{+} \in \wedge^{k_{+}-i+1} H_{+}$, one has, using the definition and the commutativity of the diagrams

$$
\begin{aligned}
\varphi\left(i_{-}\left(u_{-}\right) \wedge i_{+}\left(w_{+}\right)\right) & =\operatorname{det}_{+}\left(\rho_{\tau}\left(u_{-}\right) \wedge w_{+}\right) \\
& =i_{\beta}(\varphi)\left(i_{+}\left(\rho_{\tau}\left(u_{-}\right)\right) \wedge i_{+}\left(w_{+}\right)\right) \\
& =\varphi\left(i_{+}\left(\rho_{\tau}\left(u_{-}\right)\right) \wedge i_{+}\left(w_{+}\right) \wedge \beta\right) .
\end{aligned}
$$

So we get

$$
i_{-}\left(u_{-}\right)=(-1)^{k_{+}-i+1} i_{+}\left(\rho_{\tau}\left(u_{-}\right)\right) \wedge \beta .
$$

It follows that $\rho_{\tau}\left(u_{-}\right)$is zero unless $u_{-}$is of the form $u_{-}^{\prime} \wedge i_{-}^{-1}(\beta)$, in which case $\rho_{\tau}\left(u_{-}\right)=(-1)^{k_{+}-i+1} i_{+}^{-1} i_{-}\left(u_{-}^{\prime}\right)$. Here, $i_{-}^{-1}(\beta)$ and $i_{+}^{-1} i_{-}\left(u_{-}^{\prime}\right)$ denote arbitrary elements of the preimage. 


\section{Plat position}

A system of $n$ disjoint arcs $A_{1}, \ldots, A_{n}$ properly embedded in $D \times I$ is called trivial if they are not linked and are boundary parallel; more precisely, if there exist $n$ disjoint disks $D_{1}, \ldots, D_{n}$ such that $A_{i} \cap D_{i}=A_{i} \subset \partial D_{i}, \partial D_{i} \backslash A_{i} \subset \partial(D \times I)$, and $A_{j} \cap D_{i}=\emptyset$, for each $i, j=1, \ldots, n$ with $i \neq j$. A trivial tangle of type $(k, n)$ is a tangle whose component are $k$ vertical strands connecting $D_{+}$with $D_{-}$and a system of $n$ trivial arcs such that if there exists an arc of $\tau$ connecting two points in $D_{+}$, then no arc of $\tau$ connects two points in $D_{-}$.

A Heegaard surface for a tangle $\tau \subset D \times I$ is a disk that intersects the tangle transversally and cuts $\tau$ into two trivial tangles.

To see the existence of a Heegaard surface for a given tangle, start with a real valued function $f$ on $D \times I$ that is of Morse type on the tangle: typically we can imagine $f$ as the height function on $D \times I$. By perturbing the function, we can assume that minima occur before maxima; so if $y=1 / 2$ is a regular value separating the minima from the maxima then $f^{-1}(1 / 2)$ is the required Heegaard disk.

If we choose a standard model $\tau(k, n)$ for a trivial tangle of type $(k, n)$, the existence of a Heegaard surface for each tangle can be rephrased in the following way: each tangle $(D \times I, \tau)$ can be obtained as

$$
\left(D \times[0,1 / 2], \tau\left(k_{-}, n_{-}\right)\right) \cup_{\sigma}\left(D \times[1 / 2,1], \tau\left(k_{+}, n_{+}\right)\right),
$$

where $\sigma$ is an automorphism of the $k_{-}+2 n_{-}=k_{+}+2 n_{+}$punctured disk $D \times$ $\{1 / 2\} \cap \tau$. Since everything can be done up to isotopy, we can think of $\sigma$ as an element of the braid group $B_{k_{-}+2 n_{-}}$. We call such a decomposition of a given tangle a Heegaard splitting of the tangle.

Lemma 4.1. Let $\tau$ be a $\left(\epsilon^{-}, \epsilon^{+}\right)$-tangle where the length of $\epsilon^{ \pm}$equals to $k_{ \pm}+1$. Then any Heegaard splitting of $\tau$, induces a presentation of $H$ with deficiency $k=\frac{k_{-}+k_{+}}{2}$.

Proof. Let

$$
(D \times I, \tau)=\left(D \times[0,1 / 2], \tau\left(k_{-}, n_{-}\right)\right) \cup_{\sigma}\left(D \times[1 / 2,1], \tau\left(k_{+}, n_{+}\right)\right)
$$

be a Heegaard splitting for $\tau$, with $\sigma \in B_{k_{-}+2 n_{-}}$. Set

$$
\begin{aligned}
& X_{1}=\left(D \times\left[0, \frac{1}{2}\right]\right) \backslash \tau\left(k_{-}, n_{-}\right), \\
& X_{2}=\left(D \times\left[\frac{1}{2}, 1\right]\right) \backslash \tau\left(k_{+}, n_{+}\right),
\end{aligned}
$$

and

$$
S=\left(D \times\left\{\frac{1}{2}\right\}\right) \backslash \tau\left(k_{-}, n_{-}\right)=D \backslash\left\{p_{0}, \ldots, p_{k_{-}+2 n_{-}}\right\} .
$$

Thus, $B_{\tau}=(D \times I) \backslash \tau=X_{1} \cup_{\sigma} X_{2}$. By applying the Mayer-Vietoris exact sequence to this decomposition we obtain the exact sequence of reduced homology groups

$$
\ldots \longrightarrow H_{1}(S) \stackrel{i_{S_{-}} \oplus i_{S_{+}}}{\longrightarrow} H_{1}\left(X_{1}\right) \oplus H_{1}\left(X_{2}\right) \longrightarrow H_{1}\left(B_{\tau}\right) \longrightarrow 0
$$

where $i_{S_{-}}$is induced by the inclusion $S \hookrightarrow X_{1}$ while $i_{S_{+}}$is induced by $S \stackrel{\sigma}{\longrightarrow}$ $\sigma(S) \hookrightarrow X_{2}$. Let $\chi: \pi_{1}\left(B_{\tau}\right) \rightarrow \mathbb{Z}$ be the epimorphism defining $H=H_{1}^{\chi}\left(B_{\tau} ; R\right)$. This exact sequence lifts to the following exact sequence of homology with coefficients in $R$. 


$$
\ldots \longrightarrow H_{1}^{\chi}(S) \stackrel{\iota_{S} \oplus \iota_{S_{+}}}{\longrightarrow} H_{1}^{\chi}\left(X_{1}\right) \oplus H_{1}^{\chi}\left(X_{2}\right) \longrightarrow H \longrightarrow 0 .
$$

This is a presentation of $H$ as an $R$-module, since $H_{1}^{\chi}(S), H_{1}^{\chi}\left(X_{1}\right)$ and $H_{1}^{\chi}\left(X_{2}\right)$ are free $R$-modules.

Since $X_{1}, X_{2}$ and $S$ have the homotopy type of a wedge of circles, the homology modules have respectively ranks $k_{-}+n_{-}, k_{+}+n_{+}$and $k_{-}+2 n_{-}$. It follows that the deficiency of the presentation is $k$.

By construction, $\rho_{\tau}$ is well-defined up multiplication by units of $R$. This implies that changing the Heegaard splitting of $\tau$ will change $\rho_{\tau}$ up to multiplication by units of $R$. Nevertheless, we give a brief constructive proof of this fact, since it illustrates the similarities with the approach of Bigelow for the Jones polynomial Bi. In order to do this we need to introduce some new definitions.

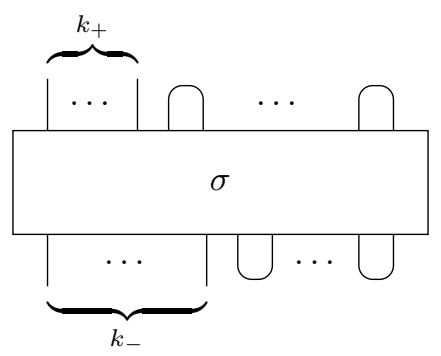

Figure 3. The $\left(k_{+}, k_{-}\right)$-plat closure of $\sigma$.

Given an oriented braid $\sigma \in B_{r}$, and integers $k_{+}, k_{-} \leq r$ of the same parity as $r$, the $\left(k_{+}, k_{-}\right)$-plat closure of $\sigma$ is the tangle obtained from $\sigma$ as follows. Place caps to connect adjacent pairs of endpoints at the top right, so as to leave only the $k_{+}$endpoints at the top left. Similarly, place cups to connect adjacent pairs of endpoints at the bottom right, so as to leave only the $k_{-}$endpoints at the bottom left (see Figure 4). Let $\hat{\sigma}\left(k_{+}, k_{-}\right)$denote the $\left(k_{+}, k_{-}\right)$-plat closure of $\sigma$. Clearly

$$
\left(D \times I, \hat{\sigma}\left(k_{+}, k_{-}\right)\right)=\left(D \times[0,1 / 2], \tau\left(k_{-}, n_{-}\right) \cup_{\sigma}\left(D \times[1 / 2,1], \tau\left(k_{+}, n_{+}\right)\right) .\right.
$$

In order to relate two braids having the same $\left(k_{+}, k_{-}\right)$-plat closure we define particular subgroups of the braid groups: let $\mathrm{Hil}_{k+2 n}$ be the subgroup of $B_{k+2 n}$ generated by the following braids

$$
\begin{array}{ll}
\text { - } \sigma_{k+1} \\
\text { - } \sigma_{k+2} \sigma_{k+1}^{2} \sigma_{k+2} \\
\sigma_{k+2 i} \sigma_{k+2 i-1} \sigma_{k+2 i+1} \sigma_{k+2 i} \text { for } i=1, \ldots n-1,
\end{array}
$$

where $\sigma_{1}, \ldots \sigma_{k+2 n-1}$ are the standard generators of $B_{k+2 n}$. When $k=0$ the above subgroups are well known and are called Hilden braid groups on $2 n$ strings (see BC].

We have the following theorem that extend to our setting a result of Birman for classical plat closure of braids (see [B2]) .

Theorem 4.2. Two oriented braids have isotopic $\left(k_{+}, k_{-}\right)$-plat closures if and only if they are related by a finite sequence the following moves: 
- $\sigma \rightarrow \sigma k$, where $\sigma \in B_{k_{-}+2 n}$ and $k \in \mathrm{Hil}_{k_{-}+2 n}$

- $\sigma \rightarrow h \sigma$, where $\sigma \in B_{k_{+}+2 n}$ and $h \in \operatorname{Hil}_{k_{+}+2 n}$

- $\sigma \leftrightarrow \sigma \sigma_{s}$, where $\sigma \in B_{s}$ and $\sigma_{s} \sigma \in B_{s+2}$.

Proof. The proofs of $\left[\mathrm{B} 2\right.$, Theorem 1 and $\left.1^{\prime}\right]$ extend to our case.

Now we are ready to state our result.

Theorem 4.3. Let $\mathcal{H}$ and $\mathcal{H}^{\prime}$ be two Heegaard splittings of the same $\left(\epsilon^{-}, \epsilon^{+}\right)$tangle $\tau$. Specifically, suppose $\mathcal{H}$ and $\mathcal{H}^{\prime}$ are respectively

$$
\begin{aligned}
& (D \times I, \tau)=\left(D \times[0,1 / 2], \tau\left(k_{-}, n_{-}\right)\right) \cup_{\sigma}\left(D \times[1 / 2,1], \tau\left(k_{+}, n_{+}\right)\right), \\
& (D \times I, \tau)=\left(D \times[0,1 / 2], \tau\left(k_{-}, m_{-}\right)\right) \cup_{\varsigma}\left(D \times[1 / 2,1], \tau\left(k_{+}, m_{+}\right)\right) .
\end{aligned}
$$

Moreover, let $\rho_{\tau}$ and $\rho_{\tau}^{\prime}$ be the module homomorphisms computed as defined in Section 2 using the presentations of $H$ associated to the two Heegaard spittings (see Lemma 4.1]. Then $\rho_{\tau}^{\prime}=m_{u} \circ \rho_{\tau}$, where $m_{u}$ denotes the multiplication by a unit of $R$.

Proof. We keep the notations of the proof of Lemma 4.1

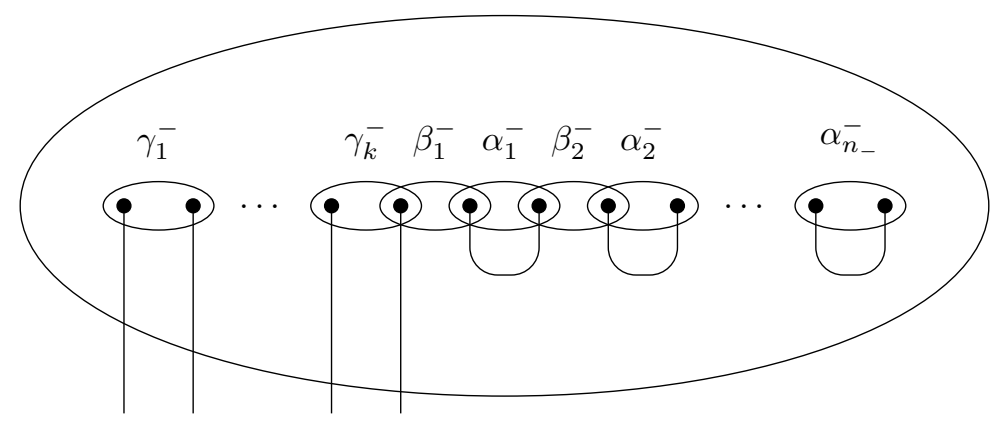

Figure 4. Curves on $S$, and the bottom half of the tangle.

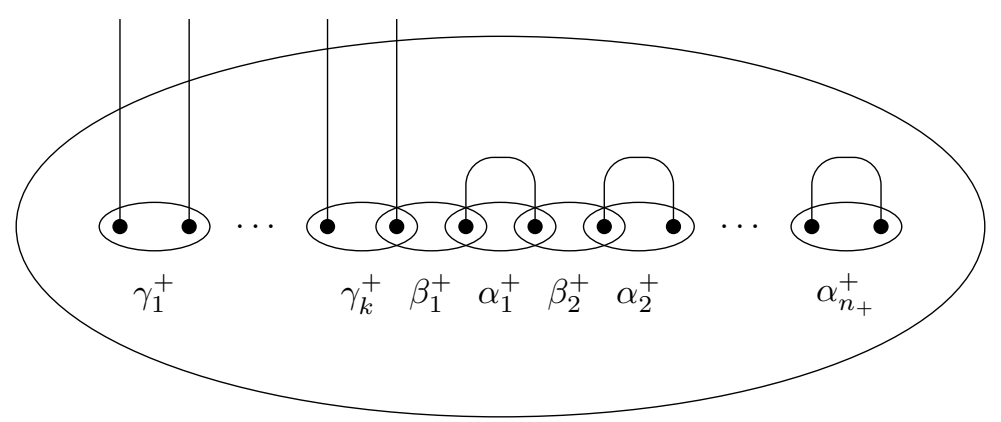

Figure 5. Curves on $S$, and the top half of the tangle.

Let $\gamma_{1}^{-}, \ldots, \gamma_{k_{-}}^{-}, \beta_{1}^{-}, \alpha_{1}^{-}, \ldots, \beta_{n_{-}}^{-}, \alpha_{n_{-}}^{-}$be the basis for $H_{1}^{\chi}(S)$ as described in Remark 2 Thus, each of these is either a loop or figure eight around two adjacent 
punctures in $S$. The $\gamma_{i}^{-}$involve two punctures connected to strands that connect to the bottom of the tangle. The $\alpha_{i}^{-}$are loops around the two endpoints of a cup, and become zero in the homology of the tangle complement since they could slide off the cup. The $\beta_{i}^{-}$involve punctures from adjacent cups, or in the case of $\beta_{1}^{-}$, a cup and a strand. See Figure 4, but there we assumed the punctures alternate in sign so as to avoid having to draw any figure eight.

Now $\gamma_{1}^{-}, \ldots, \gamma_{k_{-}}^{-}, \beta_{1}^{-}, \ldots, \beta_{n_{-}}^{-}$is a basis for the free $R$-module $H_{1}^{\chi}\left(X_{1}\right)$, and $\iota_{S_{-}}$ is a projection with kernel generated by the $\alpha_{i}^{-}$. Moreover, $\gamma_{1}^{-}, \ldots, \gamma_{k_{-}}^{-}$is a basis for the free $R$-module $H_{-}=H_{1}^{\chi-}\left(D_{-} ; R\right)$.

Let $\gamma_{1}^{+}, \ldots, \gamma_{k_{+}}^{+}, \beta_{1}^{+}, \ldots, \beta_{n_{+}}^{+}, \alpha_{1}^{+}, \ldots, \alpha_{n_{+}}^{+}$be the lifts of the loops of Figure 5 . Then $\gamma_{1}^{+}, \ldots, \gamma_{k_{+}}^{+}, \beta_{1}^{+}, \ldots, \beta_{n_{+}}^{+}$is a basis for the free $R$-module $H_{1}^{\chi}\left(X_{2}\right)$. Note that $k_{-}+2 n_{-}=k_{+}+2 n_{+}$.

Let $\left(f_{1}, \ldots, f_{k_{+}}\right)$be a ordered basis of $H_{+}$as a free $R$-module and let

$$
\operatorname{det}_{+}: \wedge^{k_{+}} H_{+} \rightarrow R
$$

be the corresponding determinant. For each sequence $I$ :

$$
1 \leq i_{1}<\cdots<i_{n} \leq k_{+}-1
$$

we set $\hat{f}_{I}=f_{i_{1}} \wedge \cdots \wedge f_{i_{n}}$. Moreover, we denote with $\bar{I}$ the sequence complementary to $I$ with respect to $1<2<\cdots<k_{+}$. If $u_{-} \in \wedge^{i} H_{-}$, then $\rho_{\tau}\left(u_{-}\right)=\sum_{I} a_{I} \hat{f}_{I} \in$ $\wedge^{i+\delta k} H_{+}$, where

$$
a_{I}=\operatorname{det}_{+}^{-1}\left(\varphi(H, k)\left(i_{-}\left(u_{-}\right) \wedge i_{+}\left(\hat{f}_{\bar{I}}\right)\right)\right)
$$

and $\varphi(H, k)$ is the Alexander function associated to the presentation of $H$ induced by $\mathcal{H}$ as in proof of Lemma 4.1 .

Let $b(\sigma)$ denote the Burau matrix of $\sigma$, using the above bases. The presentation matrix of $H$ with respect to the basis $\left(\gamma_{i}^{-}, \beta_{j}^{-}, \alpha_{j}^{-}\right)$of $H_{1}^{\chi}(S)$ and $\left(\gamma_{i}^{-}, \beta_{j}^{-}, \gamma_{h}^{+}, \beta_{l}^{+}\right)$ of $H_{1}^{\chi}\left(X_{1}\right) \oplus H_{1}^{\chi}\left(X_{2}\right)$ is

$$
\left(\begin{array}{ccc}
I_{k_{-}} & 0 & 0 \\
0 & I_{n_{-}} & 0 \\
b(\sigma)\left(\gamma^{-}\right)_{\mid \gamma^{+}} & b(\sigma)\left(\beta^{-}\right)_{\mid \gamma^{+}} & b(\sigma)\left(\alpha^{-}\right)_{\mid \gamma^{+}} \\
b(\sigma)\left(\gamma^{-}\right)_{\mid \beta^{+}} & b(\sigma)\left(\beta^{-}\right)_{\mid \beta^{+}} & b(\sigma)\left(\alpha^{-}\right)_{\mid \beta^{+}}
\end{array}\right)
$$

where the terms of the matrix are blocks.

As a basis of $H_{-}$(respectively $\left.H_{+}\right)$we can choose the lifts of the loops $\left(\gamma_{1}^{-}, \ldots, \gamma_{k_{+}}^{-}\right)$ (respectively $\left(\gamma_{1}^{+}, \ldots, \gamma_{k_{+}}^{+}\right)$) depicted in Figure 4 and 5 , so we just have to check how the matrix used to compute $\varphi(H, k)\left(i_{-}\left(\hat{\gamma}_{I}^{-}\right) \wedge i_{+}\left(\hat{\gamma}_{J}^{+}\right)\right)$changes under the moves of Theorem 4.2 Following the definition of Alexander function and the computation of Lemma 4.1] we have that $\varphi(H, k)\left(i_{-}\left(\hat{\gamma}_{I}^{-}\right) \wedge i_{+}\left(\hat{\gamma}_{J}^{+}\right)\right)$is the determinant of the following matrix

$$
M=\left(\begin{array}{ccccc}
I_{k_{-}} & 0 & 0 & E_{I}^{-} & 0 \\
0 & I_{n_{-}} & 0 & 0 & 0 \\
b(\sigma)\left(\gamma^{-}\right)_{\mid \gamma^{+}} & b(\sigma)\left(\beta^{-}\right)_{\mid \gamma^{+}} & b(\sigma)\left(\alpha^{-}\right)_{\mid \gamma^{+}} & 0 & E_{J}^{+} \\
b(\sigma)\left(\gamma^{-}\right)_{\mid \beta^{+}} & b(\sigma)\left(\beta^{-}\right)_{\mid \beta^{+}} & b(\sigma)\left(\alpha^{-}\right)_{\mid \beta^{+}} & 0 & 0
\end{array}\right),
$$

where

$$
\left(E_{I}^{-}\right)_{h k}=\left\{\begin{array}{ll}
1 & \text { if } k=i_{h} \\
0 & \text { otherwise }
\end{array} \quad \text { and } \quad\left(E_{J}^{+}\right)_{h k}= \begin{cases}1 & \text { if } k=j_{h} \\
0 & \text { otherwise }\end{cases}\right.
$$


So we want to check how the determinant of the matrix $M$ changes under the moves of Theorem 4.2

The last move changes the braid index by two. Let $N=k_{-}+2 n_{-}$and suppose that $\sigma \in B_{N}$. Then $\varsigma=\sigma \sigma_{N} \in B_{N+2}$. Moreover, by the functoriality of the Burau representation, $b\left(\sigma \sigma_{N}\right)=b(\sigma) b\left(\sigma_{N}\right)$. An explicit computation gives the presentation matrix of $H$, relatively to the Heegaard decomposition $\mathcal{H}^{\prime}$ :

$$
P^{\prime}=\left(\begin{array}{ccccc}
I_{k_{-}} & 0 & 0 & 0 & 0 \\
0 & I_{n_{-}} & 0 & 0 & 0 \\
0 & 0 & 1 & 0 & 0 \\
b(\sigma)\left(\gamma^{-}\right) \mid \gamma^{+} & b(\sigma)\left(\beta^{-}\right)_{\mid \gamma^{+}} & 0 & b(\sigma)\left(\alpha^{-}\right)_{\mid \gamma^{+}} & \\
b(\sigma)\left(\gamma^{-}\right)_{\mid \beta^{+}} & b(\sigma)\left(\beta^{-}\right)_{\mid \beta^{+}} & 0 & b(\sigma)\left(\alpha^{-}\right)_{\mid \beta^{+}} & 0 \\
0 & 0 & -t^{\epsilon+1} & 0 \ldots 0 t^{\epsilon+1} & 1
\end{array}\right),
$$

with respect to the bases

$$
\begin{gathered}
\left(\gamma_{i}^{-}, \beta_{j}^{-}, \beta_{n_{-}+1}^{-}, \alpha_{j}^{-}, \alpha_{n_{-}+1}\right), \\
\left(\gamma_{i}^{-}, \beta_{j}^{-}, \beta_{n_{-}+1}^{-}, \gamma_{h}^{+}, \beta_{l}^{+}, \beta_{n_{+}+1}^{+}\right) .
\end{gathered}
$$

It is straightforward to check that the Alexander function associated to this presentation matrix is $\pm \operatorname{det} M$.

The first two moves of Theorem 4.2 do not change the braid index of $\sigma$ and so the size of $M$. So it is enough to check how the matrix $M$ changes by composing $\sigma$ (on the left or on the right) with a generator of $\mathrm{Hil}_{k+2 n}$. We leave the detail of the computations to the reader.

\section{Functoriality}

In this section, we prove that the Alexander invariant defined in Section 2 and computed on cups, caps and braids, in Section 3, is a functorial invariant of the tangle category.

Lemma 5.1. Let $\tau$ be a tangle in plat position. The Alexander invariant of $\tau$ from Section 4 is the same as the Alexander invariant computed as a product of cups, a braid and caps from Section 3 .

Proof. Fix a tangle $\tau$, which consists of a collection of cups at the bottom, a collection of caps at the top, and a braid $\sigma$ in the middle.

Let $\gamma_{i}^{-}$be the generators of the homology of the bottom disk of $\tau$, as shown in Figure 4. Fix $\gamma_{I}^{-}$, a wedge product of a sequence of these basis vectors.

Let $\gamma_{j}^{+}$be the generators of the homology of the top disk of $\tau$, as shown in Figure 5. Fix $\gamma_{\bar{J}}^{-}$, a wedge product of a sequence of these basis vectors, where $\bar{J}$ is the complement of $J$. We will compute the coefficient of $\gamma_{\bar{J}}^{-}$in $\rho(\tau)\left(\gamma_{I}^{-}\right)$.

Let $\alpha_{i}^{-}$be the loops around the cups, as shown in Figure 4. Let $\alpha^{-}$be the wedge product of all of these elements in order. If we apply just the cups from $\tau$ to $\gamma_{I}^{-}$, we obtain $\gamma_{I}^{-} \wedge \alpha^{-}$. Now apply the braid $\sigma$, to obtain $b(\sigma)\left(\left(\gamma_{I}^{-}\right) \wedge\left(\alpha^{-}\right)\right)$.

Let $\beta_{i}^{+}$be the loops that go between adjacent caps of $\tau$, as shown in Figure 5 . Let $\beta^{+}$be the wedge product of all of these elements in order. The coefficient of $\gamma_{\bar{J}}^{-}$in $\rho(\tau)\left(\gamma_{I}^{-}\right)$is the coefficient of $\gamma_{\bar{J}}^{-} \wedge \beta^{+}$in $b(\sigma)\left(\gamma_{I}^{-}\right) \wedge b(\sigma)\left(\alpha^{-}\right)$. 
Now we recompute this same coefficient. Using notation from Section 4, the coefficient of $\gamma_{\bar{J}}^{-}$in $\rho(\tau)\left(\gamma_{\bar{I}}^{-}\right)$is the determinant of:

$$
\left(\begin{array}{ll}
E_{\bar{J}}^{+} & E_{J}^{+}
\end{array}\right)
$$

times the determinant of:

$$
\left(\begin{array}{ccccc}
I_{k_{-}} & 0 & 0 & E_{I}^{-} & 0 \\
0 & I_{n_{-}} & 0 & 0 & 0 \\
b(\sigma)\left(\gamma^{-}\right)_{\mid \gamma^{+}} & b(\sigma)\left(\beta^{-}\right)_{\mid \gamma^{+}} & b(\sigma)\left(\alpha^{-}\right)_{\mid \gamma^{+}} & 0 & E_{J}^{+} \\
b(\sigma)\left(\gamma^{-}\right)_{\mid \beta^{+}} & b(\sigma)\left(\beta^{-}\right)_{\mid \beta^{+}} & b(\sigma)\left(\alpha^{-}\right)_{\mid \beta^{+}} & 0 & 0
\end{array}\right) .
$$

The first of these determinants is \pm 1 , which we will deal with later. The second matrix will be simplified using column operations that do not change the determinant. Specifically, use the left column of blocks to cancel out the $E_{I}^{-}$in the fourth column of blocks. This gives the following.

$$
\left(\begin{array}{ccccc}
I_{k_{-}} & 0 & 0 & 0 & 0 \\
0 & I_{n_{-}} & 0 & 0 & 0 \\
b(\sigma)\left(\gamma^{-}\right)_{\mid \gamma^{+}} & b(\sigma)\left(\beta^{-}\right)_{\mid \gamma^{+}} & b(\sigma)\left(\alpha^{-}\right)_{\mid \gamma^{+}} & -b(\sigma)\left(\gamma_{I}^{-}\right)_{\mid \gamma^{+}} & E_{J}^{+} \\
b(\sigma)\left(\gamma^{-}\right)_{\mid \beta^{+}} & b(\sigma)\left(\beta^{-}\right)_{\mid \beta^{+}} & b(\sigma)\left(\alpha^{-}\right)_{\mid \beta^{+}} & -b(\sigma)\left(\gamma_{I}^{-}\right)_{\mid \beta^{+}} & 0
\end{array}\right) .
$$

This has the same determinant as the smaller matrix from its bottom right.

$$
\left(\begin{array}{llc}
b(\sigma)\left(\alpha^{-}\right)_{\mid \gamma^{+}} & -b(\sigma)\left(\gamma_{I}^{-}\right)_{\mid \gamma^{+}} & E_{J}^{+} \\
b(\sigma)\left(\alpha^{-}\right)_{\mid \beta^{+}} & -b(\sigma)\left(\gamma_{I}^{-}\right)_{\mid \beta^{+}} & 0
\end{array}\right) .
$$

Up to sign, this is the same as the determinant of:

$$
\left(\begin{array}{ccc}
b(\sigma)\left(\gamma_{I}^{-}\right)_{\mid \gamma^{+}} & E_{J}^{+} & b(\sigma)\left(\alpha^{-}\right)_{\mid \gamma^{+}} \\
b(\sigma)\left(\gamma_{I}^{-}\right)_{\mid \beta^{+}} & 0 & b(\sigma)\left(\alpha^{-}\right)_{\mid \beta^{+}}
\end{array}\right)
$$

Here we switched two block columns and changed the sign of one. This may change the sign of the determinant, depending on the parity of the number of basis vectors in $\gamma_{I}^{-}$. We can ignore this, since we are free to consistently change the sign of every entry of the matrix $\rho(\tau)$.

Finally, observe that the left and right columns of the above matrix represent the terms of $b(\sigma)\left(\gamma_{I}^{-}\right) \wedge b(\sigma)\left(\alpha^{-}\right)$that are wedge products of vectors from $\gamma^{+}$and $\beta^{+}$. The determinant picks out the terms that involve precisely the vectors $\gamma_{\bar{J}}^{+}$and $\beta^{+}$. Such terms are, up to sign, $\gamma_{\frac{}{J}} \wedge \beta^{+}$. The sign correction is the determinant of

$$
\left(\begin{array}{ccc}
E_{\bar{J}}^{+} & E_{J}^{+} & 0 \\
0 & 0 & I
\end{array}\right)
$$

We conclude that this direct calculation using the methods of Section 4 gave the same answer as composing the operations of cup, braid, and cap.

Theorem 5.2. The Alexander invariant as computed in Section 3 on cups braid and caps determines a functorial invariant of the tangle category.

Proof. A presentation of the tangle category by generators and relations can be found in $[\mathrm{T}]$. Other presentations appear in the literature, sometimes with minor differences. Certainly cups, caps, and generators of the braid groups are enough to generate the tangle category. By the previous lemma, we have every relation that equates different plat presentations for the same tangle. These, together with some simple commutativity relations, are enough to give defining relations for the tangle category. 


\section{REFERENCES}

[A] Alexander J.W., Topological invariants of knots and links, Trans. Amer. Math. Soc. 30 (1928), no. 2, 275-306.

[BC] Bellingeri P.,Cattabriga A., Hilden braid groups, preprint (2009), arXiv:0909.4845.

[Bi] Bigelow S., A homological definition of the Jones polynomial, Geom. Topol. Monogr., 4, Geom. Topol. Publ., Coventry, 2002.

[B1] Birman J. S., Braids, links, and mapping class groups, Princeton University Press, Princeton, N.J. (1974), Annals of Mathematics Studies, No. 82.

[B2] Birman J. S., On the stable equivalence of plat representations of knots and links, Can. J. Math. 28 (1976), no. 2, 264-290.

[B] Burau W., Uber Zopfgruppen und gleischsinning verdrillte Verkettungen, Abh. Math. Sem. Ham. II (1936), 171-178.

[B2] Birman J.S., On the stable equivalence of plat representations of knots and links, Canad. J. Math. 28 (1976) 264-290

[CT1] Cimasoni D., Turaev V., A Lagrangian representation of tangles., Topology 44 (2005), no. $4,747-767$.

[CT2] Cimasoni, D.; Turaev, V. A Lagrangian representation of tangles. II., Fund. Math. 190 (2006), 11-27.

[FM] Florens V., Massuyeau G. Alexander functor of 3-manifolds, preprint.

[KLW] Kirk P., Livingston C., Want Z., The Gassner representation for string links, Commun. Contemp. Math. 3 (2001), no. 1, 87-136.

[La] Lawrence, R. J. A functorial approach to the one-variable Jones polynomial, J. Differential Geom. 37 (1993), no. 3, 689-710.

[Le] Lescop, C. A sum formula for the Casson-Walker invariant, Invent. Math. 133 (1998), no. 3, 613-681.

[Ld] Le Dimet J.Y., Enlacements d'intervalles et torsion de Whitehead, Bull. Soc. Math. France 129 (2001), no. 2, 215-235.

[T] Turaev, V. G. Quantum invariants of knots and 3-manifolds, de Gruyter Studies in Mathematics, 18. Walter de Gruyter and Co., Berlin, 1994.

Department of Mathematics, University of California at Santa Barbara, Santa BarBARA, CA 93106, USA

E-mail address: bigelow@math.ucsb.edu

$U R L:$ http://www.math.ucsb.edu/ ${ }^{\text {bigelow/ }}$

Dipartimento di Matematica, Piazza di Porta S. Donato 5, 40126, Bologna, Italia

E-mail address: alessia.cattabriga@unibo.it

$U R L:$ http://www.dm.unibo.it/ cattabri/

Laboratoire de Mathématiques et de leurs Applications, Pau UMR CNRS 5142, France

E-mail address: vincent.florens@univ-pau.fr

$U R L:$ http://riemann.unizar.es/geotop/pub/vincent/ 